\title{
Theoretical breakage mechanics and experimental assessment of stresses surrounding piles penetrating into dense silica sand
}

\author{
C. ZHANG*, Z. X. YANG†, G. D. NGUYEN†, R. J. JARDINE§ and I. EINAVף
}

\begin{abstract}
This letter extends an earlier theoretical and numerical analysis that aimed to improve predictions for the large-displacement end-bearing capacity of piles in sand by considering the boundary conditions applying to steady penetration and the effects of grain crushing via breakage mechanics. The earlier work led to good agreement with the end-bearing capacities, soil displacements and evolution of grain size distributions observed in experiments of penetrating model piles. The purpose of the current paper is to complement the previous evaluation through comparisons with more recently published calibration chamber measurements of the stresses surrounding instrumented model piles penetrating into dense silica sand. Encouraging agreement is demonstrated for the radial, circumferential and vertical stress components. Scope for further improvements is also identified.
\end{abstract}

KEYWORDS: particle crushing/crushability; piles; sands; stress analysis

ICE Publishing: all rights reserved

\section{NOTATION}

$B \quad$ breakage internal variable

$E_{\text {c }} \quad$ critical breakage energy

$G \quad$ shear modulus

$h \quad$ height above pile tip (positive) or depth below pile

tip (negative)

$M \quad$ friction coefficient

$q_{\mathrm{c}} \quad$ cone penetration test resistance

$R \quad$ pile radius

$r \quad$ radius of point from pile axis

$S \quad$ pile penetration depth

$z \quad$ depth below sand surface

$\varphi^{\prime} \quad$ effective angle of shearing resistance

$\sigma_{r}^{\prime} \quad$ effective radial stress; $\sigma_{r \mathrm{~m}}^{\prime}$ is moving value

$\sigma_{z}^{\prime} \quad$ effective vertical stress; $\sigma_{z \mathrm{~m}}^{\prime}$ is moving value

$\sigma_{\theta}^{\prime} \quad$ effective circumferential stress; $\sigma_{\theta \mathrm{m}}^{\prime}$ is moving value

\section{INTRODUCTION}

Better knowledge of how soil stresses vary around displacement piles is vital to understanding their highly non-linear behaviour and improving predictive capabilities for design (Jardine, 2013). Following observations of intense grain breakage during calibration chamber model pile penetration in sand (Yang et al., 2010), Zhang et al. (2013) reported a theoretical investigation into its potential effects that applied the breakage mechanics theory

Manuscript received 16 October 2013; first decision 27 November 2013; accepted 9 December 2013.

Published online at www.geotechniqueletters.com on 24 January 2014.

${ }^{*}$ GHD, Sydney, NSW, Australia

†Key Laboratory of Soft Soils and Geoenvironmental Engineering of Ministry of Education, Research Centre of Offshore and Urban Geotechnical Engineering, Department of Civil Engineering, Zhejiang University, Hangzhou, China

$\$$ School of Civil, Environmental and Mining Engineering, The University of Adelaide, Adelaide, SA, Australia

$\S$ Department of Civil and Environmental Engineering, Imperial College, London, UK

TSchool of Civil Engineering, The University of Sydney, Sydney, NSW, Australia proposed by Einav (2007a, 2007b, 2007c, 2007d) and developed by Nguyen \& Einav (2009, 2010), Rubin \& Einav (2011), Das et al. (2011) and Buscarnera \& Einav (2012). A simple breakage model led to predictions that Zhang et al. (2013) compared with internal deformation patterns and end-bearing capacities measured in model pile tests by Kuwajima et al. (2009), as well as evolving grain size distributions (GSDs) from the model tests reported by Yang et al. (2010). Figure 1 illustrates the latter case, reproducing the tip resistance-penetration depth $\left(q_{\mathrm{c}}-S\right)$ profile measured as a $36 \mathrm{~mm}$ diameter cone-ended pile penetrated into dense Fontainebleau NE34 sand, while Fig. 2 compares the post-penetration GSD curves defined in three zones around the pile with theoretical predictions. Further aspects of the latter experiments have been reported by Jardine et al. (2013a, 2013b), who conducted careful comprehensive measurements of the stress distributions developed on and around an instrumented pile. Yang et al. (2014) offer a broad review of how these data compare with related predictions made with a range of theoretical approaches and also of data gathered in other experimental studies.

This letter sets out detailed predictions made using the approach of Zhang et al. (2013) for the calibration chamber stress measurements of Jardine et al. (2013a, 2013b). The new stress predictions were made without altering the breakage mechanics model parameters or finite-element (FE) analytical procedures reported by Zhang et al. (2013). The letter also offers a brief comparative commentary that suggests ways in which the predictive approach could be developed to capture further features of the observed system response with still greater fidelity.

\section{BREAKAGE MECHANICS AND NUMERICAL ANALYSES}

Features of breakage mechanics

The aspects of breakage mechanics theory relevant to this letter are as follows.

- A continuum framework for deriving thermodynamically admissible constitutive models that relates stresses and strain rates to the evolving GSDs developed during the breakage process (comminution). 


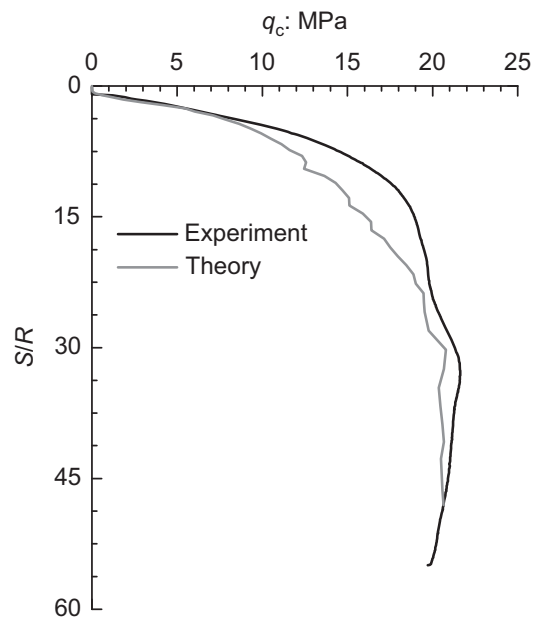

Fig. 1. End-bearing capacity $q_{c}$ versus penetration depth $S$ normalised by pile radius $R$ (i.e. $S / R$ ); replotted from Zhang et al. (2013); calibration chamber experiments from Yang et al. (2010)

- Adopting the GSD as the basis for statistical homogenisation. The GSD is evaluated using a breakage internal variable $B$ as a linear scalar term that maps the evolution of the GSD, $F(x, B)$, from an initial profile ( $B=0$, corresponding to $\left.F_{0}(x)\right)$ towards an ultimate state $\left(B=1\right.$, corresponding to $\left.F_{\mathrm{u}}(x)\right)$ (Fig. $3 ; x$ is the grain size)

$F(x, B)=(1-B) F_{0}(x)+B F_{\mathrm{u}}(x)$,

- Helmholtz free energy defined by how the stored elastic energy scales within the grains according to their surface area.

- The statistical homogenisation and the scaling of stored energy unfolds an additional geometrical parameter, $\theta$, which is an index of the soil grading. This is not a mechanical parameter and depends only on the current GSD.

- A formulation that predicts the GSD evolution of sands under varying stress and strain paths (Einav, 2007a, 2007b) by equating energy dissipation to the rate of change of the residual breakage energy (Einav, 2007c). The critical comminution pressures are connected to the GSD, and therefore the nominal grains' overall surface area, through a dependence that is analogous to that applying to the critical tensile strength of near-continuous solids in fracture mechanics.

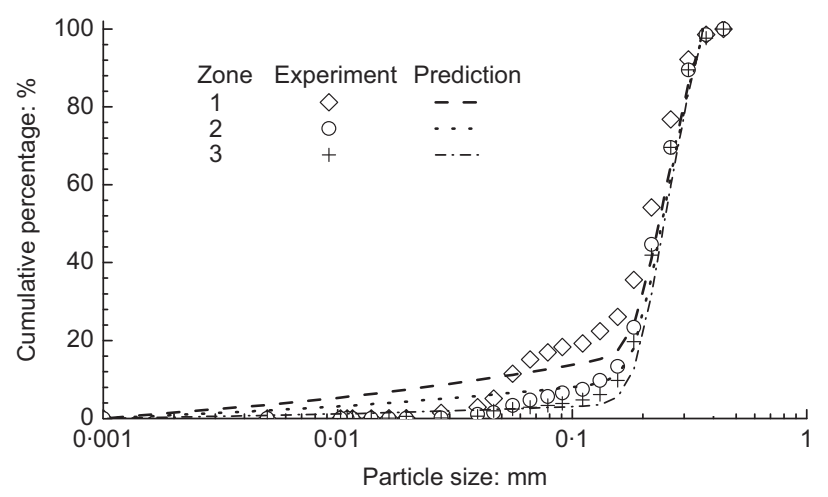

Fig. 2. GSD profiles in three grain breakage zones as defined by Yang et al. (2010) around the pile shaft, replotted from Zhang et al. (2013). Zone definitions and experimental trends from Yang et al. (2010)

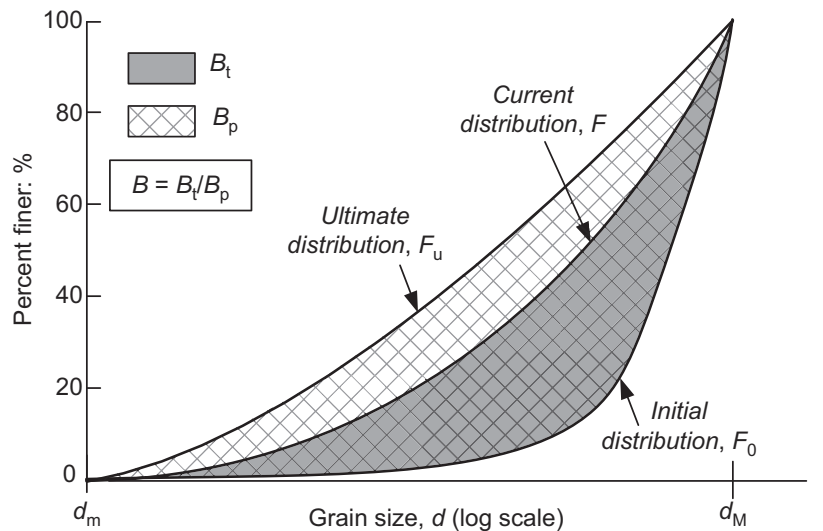

Fig. 3. Einav's breakage definition (Einav, 2007a)

For the current evaluation, the simplest breakage mechanics model was employed, which considers that the stored energy increases quadratically with the elastic strains. As such, the model requires only four mechanical parameters - two isotropic elastic stiffnesses (shear $G$ and bulk moduli $K$ ), a friction coefficient $M$ and the critical breakage energy $E_{\mathrm{c}}$, which can be obtained from conventional laboratory tests, such as isotropic tests followed by drained triaxial shear.

The same theory has been applied to confined comminution problems found in saturated and unsaturated geomechanics, geophysics, geology and mineral processing engineering (see Nguyen \& Einav, 2009, 2010; Das et al., 2011; Rubin \& Einav, 2011; Buscarnera \& Einav, 2012; Zhang et al., 2013).

\section{Numerical simulation of cone-ended displacement pile test}

Zhang et al. (2013) described the FE numerical modelling procedure (implemented in Abaqus) adopted to simulate the cone-ended pile experiments described by Yang et al. (2010) and Jardine et al. (2013a, 2013b). The axi-symmetric
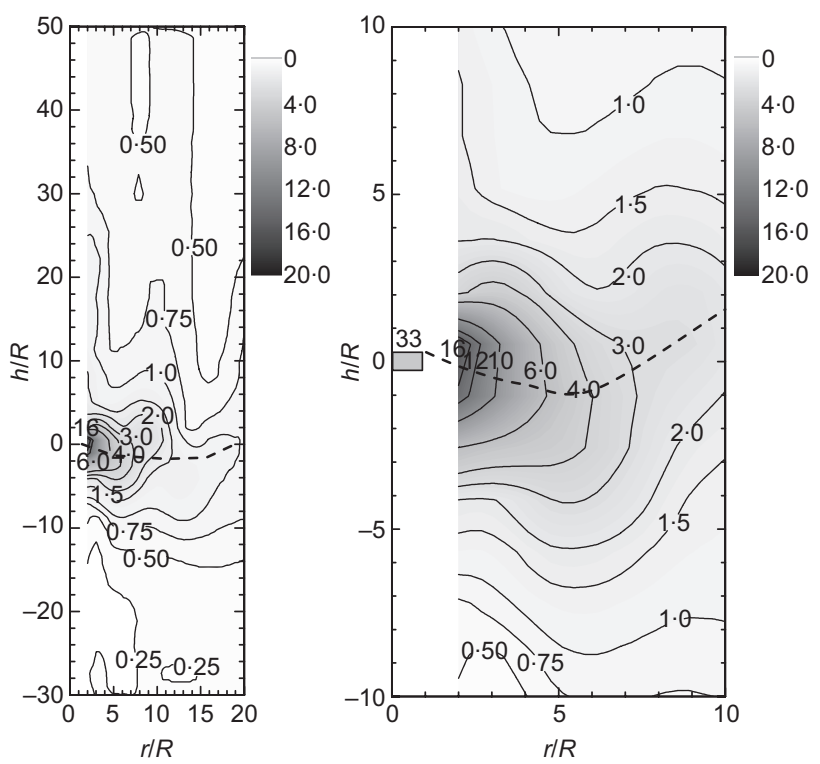

Fig. 4. Radial 'moving' stress contours during installation shown at two scales, normalised by $q_{c}$, shown as percentage. The dashed curves show locus connecting maxima developed in each case; after Jardine et al. (2013b) 


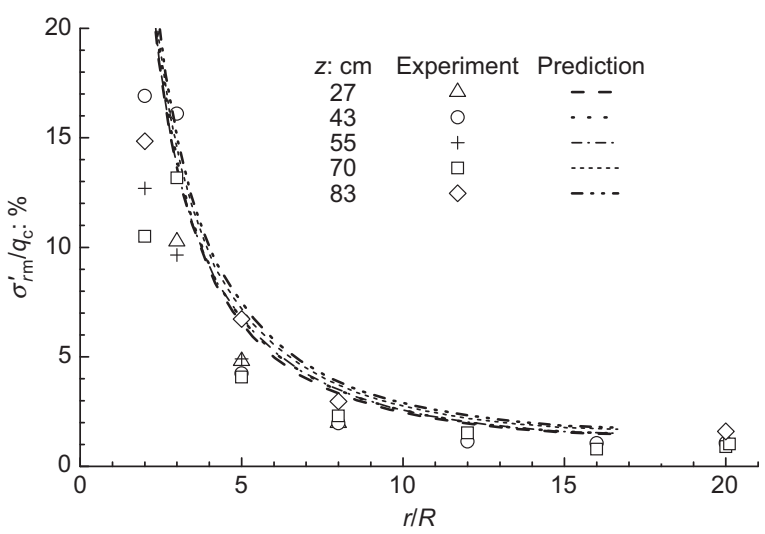

Fig. 5. Comparison of radial stress evolutions at the $h=0.5 R$ level above the tip during penetration; experimental results from Jardine et al. (2013b)

simulations followed the experimental calibration chamber procedure reported by Yang et al. (2010), except that steady penetration was used in the simulation rather than the cyclic jacking employed in the physical tests. The numerical simulations adopted an arbitrary Lagrangian-Eulerian (ALE) method to deal with the large deformations associated with steady penetration, and the simple breakage constitutive model was coded as a user subroutine in Abaqus to deliver updated stresses and breakage increments to the general FE model.

\section{STRESS DISTRIBUTION: COMPARISON OF MODEL PREDICTION AND EXPERIMENTS}

An important point to emerge from the physical experiments was a considerable difference between the 'moving' stress regime measured as the pile was penetrating and the lower 'stationary' stresses recorded during the 'zero-load' pause periods included in each jacking cycle (Jardine et al., 2013a, 2013b). The current numerical analysis models steady displacement-controlled penetration and the evaluation considers only the 'moving' stage data reported by Jardine et al. (2013a, 2013b).

\section{Radial stresses developed as pile tip advances}

Jardine et al. (2013b) summarised the stress conditions developed around penetrating piles with contour plots (such as that shown in Fig. 4), which synthesised thousands of individual stress measurements made at a range of stationary depths in the sand mass and at three moving levels on the shaft, as well as the pile tip stresses. Jardine et al. (2013b) provided comparable plots for the vertical and circumferential stresses developed during both steady penetration and pause periods. The plots adopted the moving pile tip as their geometrical origin and the stresses were normalised by the local cone resistances $q_{\mathrm{c}}$ to allow for variations with depth inside the calibration chamber. Measurements made over the shallowest section of the chamber (which was affected by the upper boundary conditions) were excluded from the synthesis. Intensive stress concentrations can be seen around the pile tip, with stresses decaying with both relative radius from the pile axis $(r / R)$ and relative height $(h / R)$ above and below the pile tip. Introducing the finite pile volume into the sand mass generally increased the radial stresses developed on the pile shaft and in the surrounding sand mass, especially at levels close to the pile tip.

Figure 5 considers the decay, with $r / R$, of the maximum normalised radial stresses observed by instruments set at five different depths $z$ below the sand surface just after the pile tip had passed each level to reach $h / R=0 \cdot 5$. The predictions of Zhang et al. (2013) are shown alongside the measurements, and it is encouraging that both indicate similarly 'exponential' decays with $r / R$ during steady penetration. Jardine et al. (2013b) showed that the profiles alter as the pile tip advances to greater depth (for higher $h / R$ values). They reported profiles of $\sigma_{r}^{\prime}$ (and $\sigma_{\theta}^{\prime}$ ) with $r / R$ that present maxima in the $2<r / R<4$ region. Jardine (2013) noted the similar trends predicted by the analysis of Zhang et al. and reviewed the important practical conclusions that follow from this key observation.

The normalised radial stress regime developed in the sand beneath and above the approaching pile tip is explored further in Fig. 6. The main trends for the soil stresses are broadly consistent between predictions and measurements over $-25<h / R<25$ and $2<r / R<8$. However, Zhang et al.'s analysis leads to higher-thanmeasured stresses at low $r / R$ and peaks at slightly lower $h / R$ values. Yang et al. (2014) highlighted a further important discrepancy. While the predicted stress regime stabilises to a steady state above $h / R=10$, the radial stress ratios $\left(\sigma_{r}^{\prime} / q_{\mathrm{c}}\right)$ measured on the pile (at $\left.r / R=1\right)$ continued to decline with $h / R$ at much higher levels. Jardine et al. (2013a, 2013b) noted that the cyclic experimental penetration

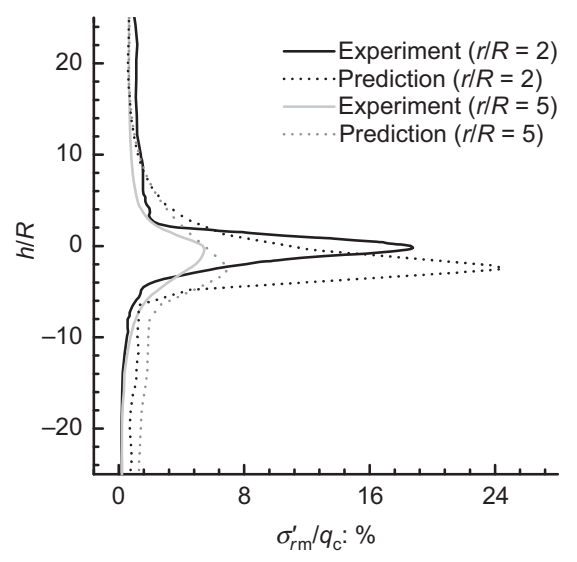

(a)

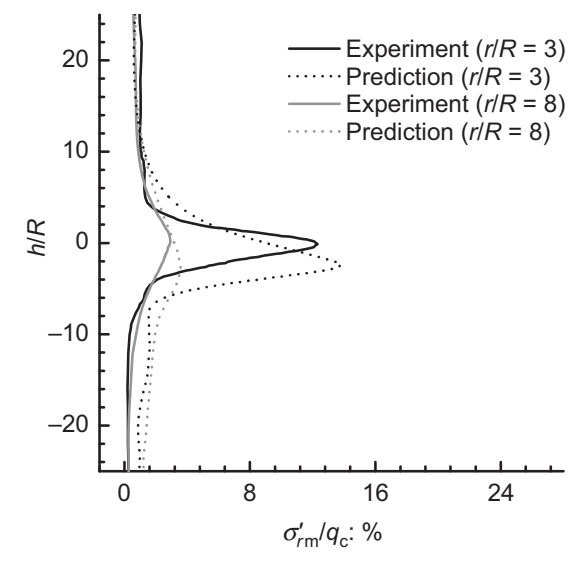

(b)

Fig. 6. Comparison of radial stresses developed as pile tip advances: (a) $r / R=2$ and $r / R=5$; (b) $r / R=3$ and $r / R=8$ 


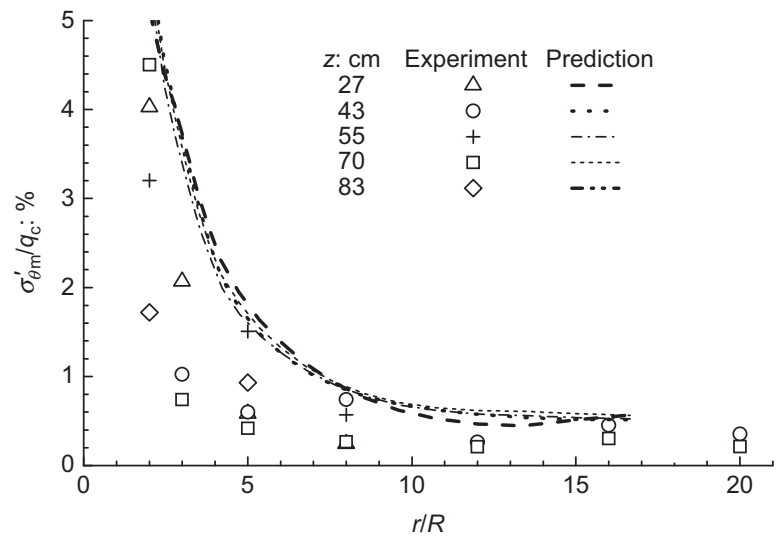

Fig. 7. Comparison of circumferential stresses at $h=0.5 R$ level above the tip during penetration; experimental results from Jardine et al. (2013b)

procedure, shaft abrasion and creep may have contributed to shaft $\sigma_{r}^{\prime} / q_{\mathrm{c}}$ ratios reducing further than expected with $h / R$. They also showed that the on-pile radial stress trends imply corresponding variations in the near-shaft $\sigma_{\theta}^{\prime} / q_{\mathrm{c}}$ profiles applying above the $h / R=10$ level.

\section{Circumferential stresses distribution}

As mentioned earlier, the model tests included circumferential stress measurements. Figure 7 presents the normalised calibration chamber measurements of circumferential stresses and profiles established at $h=0 \cdot 5 R$ in the steady penetration stages. Here, the predictions tend to show fair agreement at low $r / R$ values, but pass above the measurements in the $3<r / R<8$ region. Jardine et al. (2013b) commented on the wide experimental range of $\sigma_{r}^{\prime} / 5<\sigma^{\prime}{ }_{\theta}<$ $\sigma_{r}^{\prime} / 3 \cdot 5$ implied by Fig. 5 and Fig. 7 , and noted that the soil mass experiences failure out to a considerable radial distance where $\sigma_{r}^{\prime} / \sigma_{\theta}^{\prime}$ depends primarily on the effective angle of shearing resistance $\phi^{\prime}$ that can be mobilised under the extreme stress conditions applying. Triaxial tests described by Altuhafi \& Jardine (2011) that followed the same very high to low stress path trajectories as the pile tests indicate a strong increase in $\phi^{\prime}$ as the soil unloads and therefore predict a marked variation of $\phi^{\prime}$ and $\sigma_{r}^{\prime} / \sigma_{\theta}^{\prime}$ with $r / R$. In comparison, the simplified model of Zhang et al. (2013) assumes a constant $M$ value that leads to $\sigma_{r}^{\prime} / \sigma_{\theta}^{\prime}$ remaining close to a value of four over the area of soil failure, and so

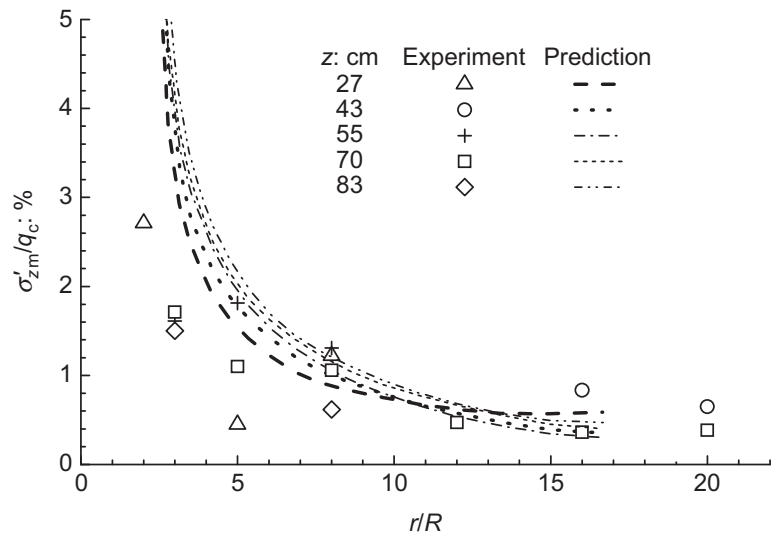

Fig. 9. Comparison of vertical stresses at $h=0.5 R$ level above tip during penetration; experimental results from Jardine et al. (2013b)

declining more gently with $r / R$ than the experimental measurements. Jardine et al. (2013b) emphasise that, as with the radial stresses, the profiles of $\sigma^{\prime}{ }_{\theta} / q_{\mathrm{c}}$ with $r / R$ have quite different shapes higher up around the pile shaft (i.e. at larger $h / R$ ).

Figure 8 compares predicted and measured $\sigma_{\theta}^{\prime} / q_{\mathrm{c}}$ trends over a wider $h / R$ range. As with the radial stresses, the predicted maxima tend to be higher than the measurements and to develop at lower $h / R$. However, the broad trends remain comparable. The experiments and FE analyses present similarly steep changes with $h / R$ and $r / R$ and, as noted above, load cycles and variations of mobilised $\phi^{\prime}$ with sand state may well explain at least part of the discrepancy seen in the mid- $r / R$ range. It is also important to recall the observations of Jardine et al. (2013a) on the difficulties of making $\sigma_{\theta}^{\prime}$ measurements, which are considered significantly less accurate than the radial stress equivalents.

\section{Vertical stresses distribution}

Finally, consider the vertical stress $\sigma_{z}^{\prime}$ predictions and measurements. Figure 9 illustrates how $\sigma_{z}^{\prime}$ varies with $r / R$ at levels near to the pile tip level $(h / R=0 \cdot 5)$. The experimental measurements scatter around the theoretical curves, revealing broadly similar trends and maximum values. As with the circumferential stresses, the measurements tend to fall below the predictions in the mid-r/R range, and this is interpreted as a further result of the steep

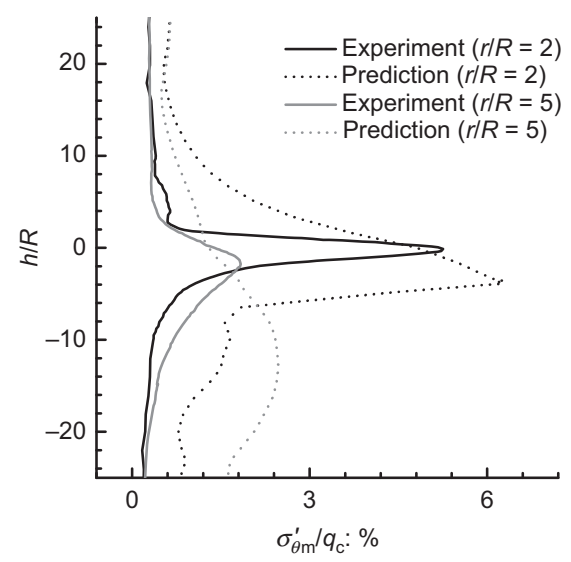

(a)

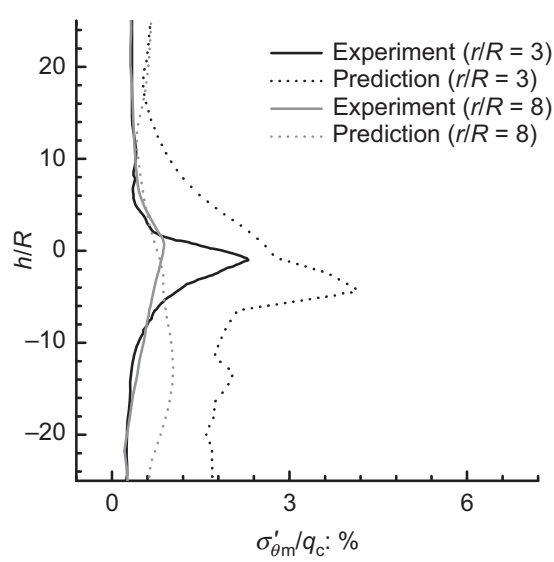

(b)

Fig. 8. Comparison of circumferential stresses developed as pile tip advances: (a) $r / R=2$ and $r / R=5$; (b) $r / R=3$ and $r / R=8$ 


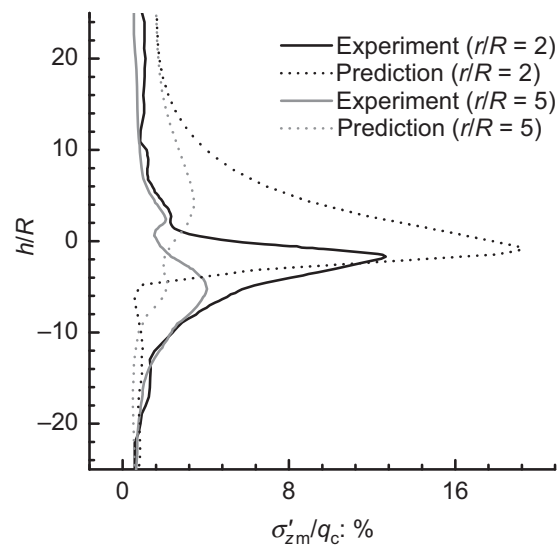

(a)

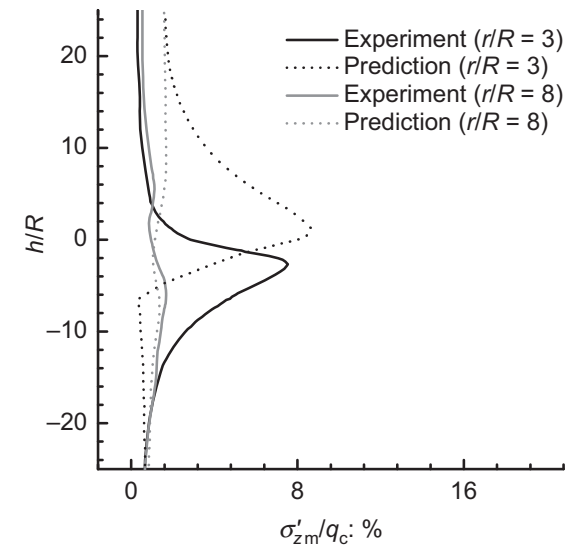

(b)

Fig. 10. Comparison of vertical stresses developed as pile tip advances: (a) $r / R=2$ and $r / R=5$; (b) $r / R=3$ and $r / R=8$

true variation in mobilised $\phi^{\prime}$ values discussed earlier. Load cycling may also have been partially influential. Overall, the vertical stresses measured at $r / R>3$ tend to fall between the (higher) $\sigma_{r}^{\prime}$ and (lower) $\sigma_{\theta}^{\prime}$ profiles. However, close to the tip (the radial region $r / R<2$ ), $\sigma_{z}^{\prime}>\sigma_{r}^{\prime}$ and comprises the largest stress. As with $\sigma_{r}^{\prime}$ and $\sigma_{\theta}^{\prime}$, differently shaped profiles apply around the shaft at higher $h / R$ values (Jardine et al., 2013b). Figure 10 presents a further comparison of the experimental and predicted trends considering a wider range of $h / R$ values. The plots also show broadly encouraging agreement, although the predicted stress maxima tend to exceed those recorded and tend to peak at slightly higher $h / R$ ratios.

\section{CONCLUSIONS}

Establishing how stresses vary around displacement piles is vital to developing better predictive capabilities for design and analysis. This letter reports how FE analysis made with a simple breakage model offered predictions for the radial, circumferential and vertical stresses developed around a conically tipped model pile during penetration into silica sand. The theoretical results and 'moving' stage experimental counterparts follow broadly similar trends and show fair quantitative agreement. Nevertheless, discrepancies are evident that suggest at least three clear avenues for further work.

- Modelling the cyclic penetration process and the response that develops after penetration halts. Marked differences were reported by Jardine et al. (2013a, 2013b) between the moving 'load' and stationary 'unload' stress profiles, which are important to understanding time effects, and residual pile load mechanisms. Adopting a breakage model variant that incorporates rate sensitivity and regularisation (as in Das et al. (2013)) offers one possible way of capturing such processes in an improved numerical model.

- Addressing shear zone abrasion and load cycling in addition to grain breakage and moving pile geometry. Laboratory and field model pile experiments (Lehane et al., 1993; Chow, 1997; Jardine et al., 2013a, 2013b) show that radial stresses developed on the pile shaft continue to decay with $h / R$ at ratios beyond the limit $h / R$ $=10$ found in the numerical simulations. Jardine et al. (2013a, 2013b) argue that this may be related to the load cycling and abrasion that is implicit in both practical jacking and pile driving.
- Applying more sophisticated constitutive models that can capture influential features such as stress state dependent failure parameters and behaviour under cyclic loading and in a representative manner. Cyclic loading phenomena can be captured by setting the breakage model within a more general and thermodynamically unified framework that adopts incrementally non-linear hypoplasticlike constitutive laws (Einav, 2012).

The analytical results are likely to depend on the assumed penetration conditions. New numerical analyses could investigate the potential effects of factors such as the initial stress field, the chamber (or field) boundary conditions and pile tip details (cone-to-flat or open-ended) as well as sand grading and grain strength. Such studies could give further valuable insights into practical issues including group interaction, bearing capacity, ageing and shaft or base stiffness.

\section{Acknowledgements}

This theoretical research was supported under Australian Research Council through the ARC Discovery Projects funding scheme (project number DP0986876). Chunshun Zhang and Giang Nguyen wish to thank the University of Sydney for the Bridging Support Grant 2013-00053 and the ARC for grant DP110102645, while ZX Yang and RJ Jardine would like to acknowledge the support from Natural Science Foundation of China (grants 51178421 and 51322809), Zhejiang University K.P. Chao's High Technology Development Foundation and the Chinese Ministry of Education Distinguished Overseas Professorship Programme and the UK Royal Society. Last but not least, the paper is dedicated to the second author's late father, Pushun Yang, for his endless love and continuous support over years.

\section{REFERENCES}

Altuhafi, F. \& Jardine, R. J. (2011). Effect of particle breakage and strain path reversal on the properties of sands located near to driven piles. Proc. 5th Int. Symp. on Deformation Characteristics of Geomaterials, Seoul 1, 386-395.

Buscarnera, G. \& Einav, I. (2012). The yielding of brittle unsaturated granular soils. Géotechnique 62, No. 2, 147-160.

Chow, F. C. (1997). Investigations into displacement pile behaviour for offshore foundations. PhD thesis, Imperial College, London, UK.

Das, A., Nguyen, G. D. \& Einav, I. (2011). Compaction bands due to grain crushing in porous rocks: a theoretical approach 
based on breakage mechanics. J. Geophys. Res. - Solid Earth 116, B08203.

Das, A., Nguyen, G. D. \& Einav, I. (2013). The propagation of compaction bands in porous rocks based on breakage mechanics. J. Geophys. Res. - Solid Earth 118, No. 5, 2049-2066.

Einav, I. (2007a). Breakage mechanics - part I: theory. J. Mech Phys. Solids 55, No. 6, 1274-1297.

Einav, I. (2007b). Breakage mechanics - part II: modelling granular materials. J. Mech. Phys. Solids 55, No. 6, 1298-1320.

Einav, I. (2007c). Fracture propagation in brittle granular matter. Proc. Royal Soc. A: Math. Phys. Engng Sci. 463, No. 2087, 3021-3035.

Einav, I. (2007d). Soil mechanics: breaking ground. Phil. Trans. Royal Soc. A: Math. Phys. Engng Sci. 365, No. 1861, 2985-3002.

Einav, I. (2012). The unification of hypo-plastic and elasto-plastic theories. Int. J. Solids Struct. 49, No. 11-12, 1305-1315.

Jardine, R. J. (2013). Advanced laboratory testing in research and practice. 2nd Bishop Lecture. Proc. ICSMGE, vol. 1. Paris: Presse des Ponts, pp. 35-55.

Jardine, R. J., Zhu, B. T., Foray, P. \& Yang, Z. X. (2013a). Measurement of stresses around closed-ended displacement piles in sand. Géotechnique 63, No. 1, 1-17.

Jardine, R. J., Zhu, B. T., Foray, P. \& Yang, Z. X. (2013b). Interpretation of stress measurements around closed-ended displacement piles in sand. Géotechnique 63, No. 8, 613-627.

Kuwajima, K., Hyodo, M. \& Hyde, A. F. (2009). Pile bearing capacity factors and soil crushability. J. Geotech. Geoenviron. Engng 135, No. 7, 901-913.

Lehane, B. M., Jardine, R. J., Bond, A. J. \& Frank, R. (1993). Mechanisms of shaft friction in sand from instrumented pile tests. J. Geotech. Engng Div. ASCE 119, No. 1, 19-35.

Nguyen, G. D. \& Einav, I. (2009). The energetics of cataclasis based on breakage mechanics. Pure Appl. Geophys. Special Issue: Mech. Struct. Evol. Fault Zones 166, No. 10-11, 1-32.

Nguyen, G. D. \& Einav, I. (2010). Nonlocal regularisation of a model based on breakage mechanics for granular materials. Int. J. Solids Struct. 47, No. 10, 1350-1360.

Rubin, M. B. \& Einav, I. (2011). A large deformation breakage model of granular materials including porosity and inelastic distortional deformation rate. Int. J. Engng Sci. 49, No. 10, $1151-1169$.

Yang, Z. X., Zhu, B. T., Jardine, R. J., Tsuha, C. H. C. \& Foray, P. (2010). Sand grain crushing and interface shearing during displacement pile installation in sand. Géotechnique 60, No. 6 , 469-482.

Yang, Z. X., Jardine, R. J., Zhu, B. T. \& Rimoy, S. (2014). A review of the stresses developed by displacement piles penetrating in sand. J. Geotech. Geoenviron. Engng 140, http://dx.doi.org/10.1061/(ASCE)GT.1943-5606.0001022.

Zhang, C., Nguyen, G. D. \& Einav, I. (2013). The end-bearing capacity of piles penetrating into crushable soils. Géotechnique 63, No. 5, 341-354.

\section{WHAT DO YOU THINK?}

To discuss this paper, please email up to 500 words to the editor at journals@ice.org.uk. Your contribution will be forwarded to the author(s) for a reply and, if considered appropriate by the editorial panel, will be published as a discussion. 